\title{
Autour de la statue de Desaix par Nanteuil, place de Jaude à Clermont-Ferrand
}

\section{Antoinette Ehrard}

\section{(2) OpenEdition \\ Journals}

\section{Édition électronique}

URL : https://journals.openedition.org/ahrf/413

DOI : $10.4000 /$ ahrf.413

ISSN : 1952-403X

\section{Éditeur :}

Armand Colin, Société des études robespierristes

Édition imprimée

Date de publication : 1 juin 2001

Pagination : 161-178

ISSN : 0003-4436

\section{Référence électronique}

Antoinette Ehrard, "Autour de la statue de Desaix par Nanteuil, place de Jaude à Clermont-Ferrand », Annales historiques de la Révolution française [En ligne], 324 | avril-juin 2001, mis en ligne le 22 mai 2006, consulté le 22 avril 2022. URL : http://journals.openedition.org/ahrf/413 ; DOI : https://doi.org/ 10.4000/ahrf.413

Ce document a été généré automatiquement le 22 avril 2022.

Tous droits réservés 


\title{
Autour de la statue de Desaix par Nanteuil, place de Jaude à Clermont- Ferrand
}

\author{
Antoinette Ehrard
}

1 À Clermont-Ferrand, le 12 août 1848, au coucher du soleil, retentit une salve de vingt et un coups de canons tirée sur la place de la Poterne, tandis que sonnent les cloches de toutes les églises. Le lendemain, au lever du soleil, est encore tirée, au même lieu, une pareille salve d'artillerie. Quel événement explique ces sonores manifestations? La proclamation de la Seconde République ? Trop tard ou trop tôt. La République avait été proclamée à Paris le 25 février 1848 ; la Constitution du 4 novembre ne le sera dans la capitale que le 12 novembre, dans les provinces le 19. Il s'agit pourtant, en août, du début de grandes cérémonies liées à la naissance du nouveau régime : l'inauguration du monument au général Desaix, sur la place de Jaude.

2 Faut-il pour autant considérer l'œuvre comme une création « révolutionnaire »? La place qu'elle tient dans la série de monuments dédiés à Desaix ainsi que l'histoire de sa commande et son style conduisent à mettre en garde contre une interprétation hâtive. La mémoire de Desaix

3 En effet, de la chute de l'Empire à l'avènement de la Seconde République la mémoire de Desaix n'est pas entièrement effacée, même sous la Restauration. La fontaine de Percier et Fortin construite en 1802 en hommage à Desaix reste en place à Paris, de même que celles de Riom et de Clermont-Ferrand ${ }^{1}$.

4 Sans doute la statue colossale élevée en 1810 par Dejoux place des Victoires ${ }^{2}$ a-t-elle été fondue en 1814, en même temps que celle de Napoléon surmontant la colonne Vendôme. Le bronze fut utilisé pour la statue équestre d'Henri IV sur le Pont-Neuf (Lemot, 1818), geste autant politique qu'économique: la statue de Desaix avait été élevée à l'emplacement de celle de Louis XIV par Desjardins, détruite en 1792.

De façon en revanche positive bien que discrète Desaix réapparaît dès 1820 dans la Galerie métallique des grands hommes français, avec une médaille de Gaunois. Sur l'avers figure son profil, avec l'inscription : «L.C. Ant. Desaix »; sur le revers : «Né à Ayat en 
MDCC.LXVIII. mort en M. DCCC Galerie métallique des grands hommes français 1820 ». Mais entre sa naissance et sa mort, ce "grand homme " n'a pas d'histoire. Trois ans plus tard, sans que la lettre fasse référence à la Révolution ni à la République, la grande gravure à l'aquatinte dessinée par Martinet, Actions glorieuses et faits d'armes du Général Desaix (30,5 x $46 \mathrm{~cm})$, sera plus explicite. Quatre moments y sont retenus : le blocus de Mayence et le passage du Rhin, en Égypte la bataille de Sediman, enfin Marengo, le 14 juin 1800. Sur la stèle qui supporte au centre le buste de Desaix on peut lire «À la postérité », référence aux dernières paroles attribuées à Desaix depuis le bulletin du 26 prairial an VIII : "Allez dire au Premier consul que je meurs avec le regret de n'avoir pas fait assez pour la postérité $»^{3}$. Faut-il ici comprendre : à Desaix, la postérité ?

Mais c'est surtout, on n'en sera pas étonné, sous la monarchie de Juillet que le texte et l'image revivifient le culte de Louis Desaix, officier du roi et général de la République. La politique de réconciliation nationale de Louis-Philippe se manifeste notamment dans les grandes commandes qui mettent l'accent sur la continuité de l'histoire de la France. Avec le nom des généraux de la République et de l'Empire, celui de Desaix est inscrit sur l'Arc de Triomphe de l'Étoile que Louis-Philippe fait achever, de 1833 à 1836. Simultanément est créé, dans l'aile du midi du château de Versailles, le musée de l'Histoire de France, inauguré en 1837. Pour la Galerie des Batailles les artistes reçoivent commande de peintures célébrant la vie militaire de la France depuis Tolbiac jusqu'au siège de la citadelle d'Anvers (23 déc. 1832). En 1835, la salle des États généraux, la salle du Sacre et de 1792, la salle des campagnes de 1793 à 1796 sont ouvertes, aboutissant à la salle de 1830. C'est pour cette dernière que Charles-Nicolas Toussaint (1732-1845) peint le grand Passage du Rhin par l'armée française à Kehl sous le commandement de Desaix et du général en chef Moreau le 24 juin 1796 (huile sur toile, 2,70 x 3,60 mètres).

7 Depuis la publication du Mémorial de Sainte-Hélène par le comte de Las Cases en 1823, l'histoire de Napoléon avait cessé d'être un sujet tabou et les compagnons de Bonaparte voient rejaillir sur eux un peu de sa gloire. C'est ainsi que dans l'Histoire de Napoléon de Laurent de l'Ardèche, éditée par Dubochet en 1838, illustrée d'après des dessins d'Horace Vernet, une lithographie (p. 97) imagine la première rencontre, en Italie, entre Bonaparte et Desaix, représentés conversant amicalement au cours d'une promenade.

8 La mémoire de Desaix n'est pas seulement honorée par des monuments ou publications nationales. Sa province natale et celles qu'il a défendues en gardent le souvenir. À l'automne 1830, autour du monument de Strasbourg élevé en 1802 par les soldats de l'armée du Rhin en mémoire de leur général ${ }^{4}$, l'Alsace lui rend un spectaculaire hommage : Hommage rendu à la mémoire des généraux Kléber et Desaix, le 10 octobre 1830, par la garnison et par la Garde nationale de Strasbourg: intermède mêlé de vers, de chants et d'évolutions militaires, par un garde national, musique de M. Demouchy, Strasbourg, imp. Silbermann, 1830.

9 L'Auvergne n'est pas en reste. Déjà, le 20 novembre 1820, la ville de Clermont-Ferrand avait donné au boulevard de la Préfecture (en 1793 «boulevard du Département ») le nom de «rue Desaix ». Dix ans plus tard, à Riom, Degeorge, architecte du Palais de justice depuis 1823, commence à construire le Château d'eau, fontaine qui aurait dû recevoir sur ses huit faces les statues de huit grands hommes de la région : Michel de l'Hospital, Chabrol, Arnauld, Pascal, Domat, Delille, Thomas et Desaix. Mais pour des raisons financières, le décor ne fut pas réalisé 5 . 
10 L'intérêt pour Desaix va se concrétiser d'abord dans les textes, moins dispendieux. En 1830, avant même les journées de Juillet, un concours avait été ouvert par la Société académique de Clermont. Les écrivains locaux étaient invités à célébrer en vers la gloire de leur compatriote. Alexandre Guillaume publiera à Paris son poème, Desaix, la Révolution et autres poésies. Élizabeth Celnart ne publiera le sien, à Clermont, que l'année suivante, dans une brochure intitulée: De l'éclectisme en littérature, suivi de Desaix et autres poésies. Essais... La même année, c'est au tour de C. A. Ravel de faire paraître, à Clermont toujours, Desaix, poème lyrique, suivi de l'anniversaire des trois journées.

11 La qualité littéraire de ces textes n'est pas notre sujet. Ce qui importe, c'est ce que manifestent ces alexandrins et octosyllabes pompeux : éloge du courage et des vertus morales dans le premier texte, référence sans honte à la révolution de 1830 dans le dernier. Le poème d'Élizabeth Celnart mérite, en dépit de sa lourdeur, d'être lu d'un peu près. L'auteur y introduit une variante dans les dernières paroles supposées du héros: "Que n'ai-je encore plus fait pour servir ma patrie» et non plus "pour la postérité ». Aucune allusion au Premier consul. En revanche, Élizabeth Celnart évoque avec enthousiasme le drapeau de la République. Décrivant les cérémonies funèbres du Grand-Saint-Bernard, elle écrit : «sur le faîte [des Alpes] / flotte d'un étendard le voile glorieux, / celui de Marengo. Gloire ! gloire immortelle, / Salut, drapeau vainqueur ! drapeau libre, salut ! / Tu fis trembler les rois [...] ». Puis faisant allusion à l'occupation de la France au retour des Bourbons et à la destruction de la statue de la place des Victoires : «L'étranger dans Paris fait la loi./ Il envahit le mont consacré par ta cendre ; / Ton bronze glorieux du socle va descendre ; / Ton bronze détrôné par l'image d'un roi... $»^{6}$. Pour conclure : "Au soleil qu'importe un nuage! / Au nom consacré d'âge en âge :/ Qu'importe le destin d'un bronze passager?». Écrits en mai 1830, ces vers pesamment mal pensants ne reçurent que le second prix, le premier étant attribué à Alexandre Guillaume.

La statue de Nanteuil

12 Bientôt va naître en Auvergne un projet plus ambitieux. Le 23 août 1838, 70 ans après la naissance de Desaix (17 août 1768), le Conseil général du Puy-de-Dôme, qui, à la première séance de la session (20 août), venait d'élire le comte Beker à sa présidence, décide l'érection d'une statue de Desaix pour la ville de Clermont ${ }^{7}$.

13 L'Auvergne possédait déjà un monument à la mémoire de Desaix, la fontaine dite la «Pyramide» de l'architecte Laurent (1801). Mais ce dernier monument était resté inachevé, puisque les quatre bas-reliefs dessinés par le sculpteur Chinard ne furent pas exécutés. La ville de Clermont ne reçut que le groupe de marbre actuellement conservé au musée d'Art Roger-Quilliot, la Mort de Desaix, qui ne figura jamais à la base de l'obélisque architecturé8.

Il s'agit ici d'une intention différente dans la mesure où le monument sera une simple effigie. Une telle initiative se place dans la continuité du culte des grands hommes instauré dès avant la Révolution. En 1776, le comte d'Angiviller avait commandé une série de statues d'hommes célèbres pour la Grande galerie du Louvre. À la Restauration, Louis XVIII projette de faire orner le pont Louis XVI (pont de la Concorde) de 12 statues de grands hommes et Louis-Philippe commande la série des bustes de grands hommes pour la Galerie historique de Versailles. Outre ces grandes commandes d'État, les sculpteurs reçoivent celles des villes qui souhaitent honorer leurs compatriotes illustres. Dans les années 1830-1840, David d'Angers crée les statues de Corneille pour 
Rouen (1831), de Gutenberg pour Strasbourg (1840), du Roi René pour Aix-en-Provence (1840), etc. Clermont-Ferrand n'est pas la ville natale de Desaix. Aussi n'est-ce pas la ville qui est à l'origine de la commande, mais le Conseil général du Puy-de-Dôme, représentatif des différentes communes du département où Desaix a vécu: Ayat-sur-Sioule où il est né, Charbonnières-les-Varennes où il a passé son enfance (Veygoux), Effiat où il fut élève de l'École royale militaire. n'était pas un inconnu. Il avait fait des débuts de peintre paysagiste puis s'était tourné vers la sculpture. Après un certain succès au Salon au début des années 1830, il venait d'exécuter les naïades et les tritons des fontaines de la place de la Concorde, achevée en 1836. La maquette conservée dans les musées de Clermont (plâtre, 62 x 49,5 x 27,5 cm.) représente, sur un cheval prêt à se cabrer, un cavalier en uniforme de général, la redingote flottant sur la croupe du cheval, tenant les rênes de la main gauche, le buste et la tête tournés vers l'arrière, le bras droit tendu qui devait brandir une épée maintenant disparue ; le visage, encadré de longs cheveux est surmonté d'un bicorne empanaché (Fig. 1). Non pas le monument purement architectural de Laurent ou d'Attiret, non pas le héros mort ou mourant de Moitte ou Chinard, ni la sublimation antiquisante de Dejoux, mais un Desaix vivant et résolument moderne.

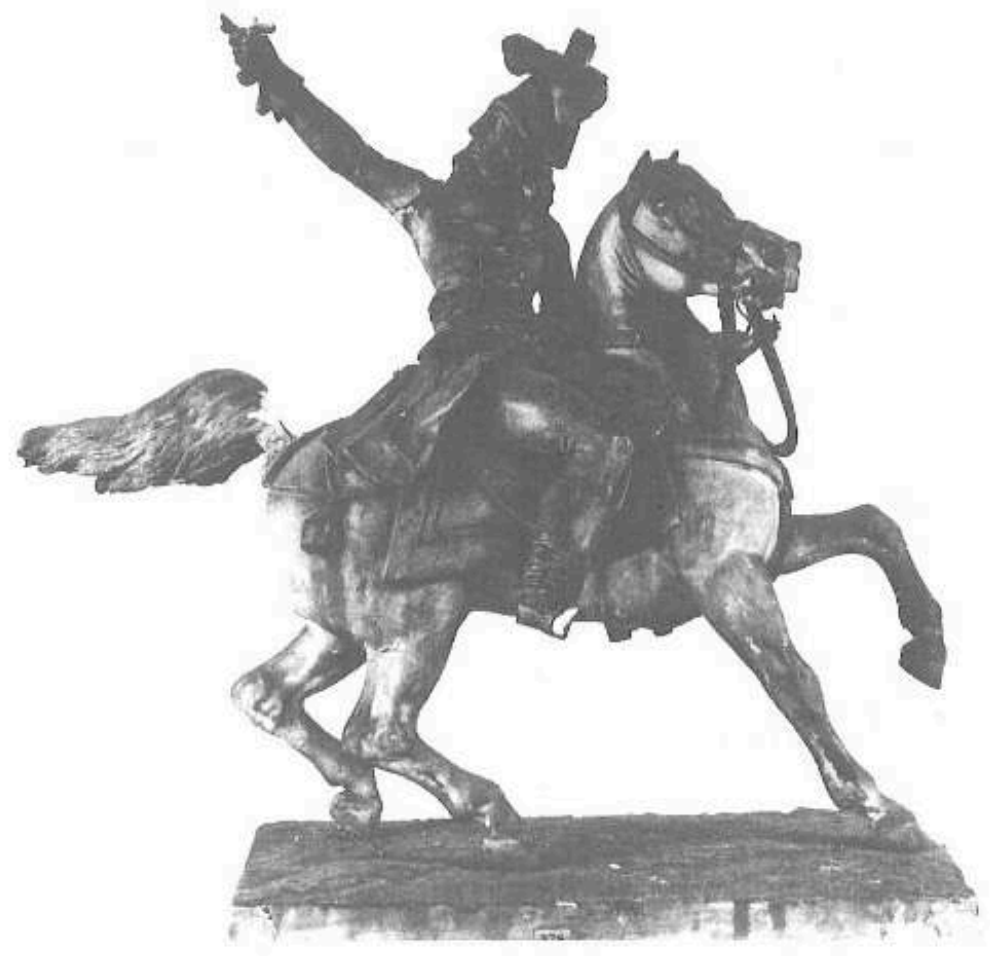

Fig. 1 - Antonin Moine,Maquette d'un monument à Desaix, plâtre, 1848. Musées de Clermont-FD

Mais, à ce projet qui ne manque pas de fougue, les commanditaires préfèrent celui du Charles-François Lebœuf, dit Nanteuil (1792-1865) ${ }^{9}$. Ce dernier, de quelques années plus âgé que Moine, jouissait d'une réputation plus ancienne. Prix de Rome de sculpture en 
1817, il s'était fait connaître au Salon en 1822, avait travaillé pour le Panthéon et l'église Notre-Dame-de-Lorette et venait d'être élu à l'Institut. La réalisation d'une statue équestre a-t-elle paru trop coûteuse ? Le prestige de l'Institut suffit-il à primer toute autre considération? Quoi qu'il en soit, le choix de cette «valeur sûre» ne fut pas une économie. Par contrat, l'artiste s'engageait à faire pour la somme de $30000 \mathrm{~F}:$ « la statue du général Desaix, de fournir le bronze et la main-d'œuvre et de faire tous les frais que nécessiterait cette statue $»^{10}$. Or, le Conseil général avait voté un crédit de 12 $000 \mathrm{~F}$ et la ville de Clermont, le 29 août, une somme de $6000 \mathrm{~F}$. Une souscription rapporte $8592 \mathrm{~F}$. Total : $26592 \mathrm{~F}$. Dans ces conditions, la commande n'est pas exécutée immédiatement. La statue ne sera achevée qu'en 1844. En 1845, pour en assurer le paiement, le duc de Morny, député du Puy-de-Dôme depuis trois ans, obtient une subvention de l'État de $3000 \mathrm{~F}$. Le bronze restera à Paris dans l'atelier de Nanteuil jusqu'en 1848.

Nanteuil fait également le choix esthétique du costume contemporain. Desaix, plus grand que nature, en uniforme, debout, dans un mouvement de marche à peine esquissé, prend appui sur la jambe droite, la jambe gauche en avant. Sa main gauche s'appuie sur son épée. Son bras droit, très peu soulevé, est tendu vers l'avant, index pointé vers le sol. Il est coiffé d'un volumineux bicorne. Un grand manteau jeté sur son épaule gauche tombe à l'arrière jusqu'au sol en plis épais, assurant l'équilibre de la statue. À ses pieds, un boulet, bien qu'il ait été tué par une balle; mais le projectile mortel doit être visible. (Fig. 2)

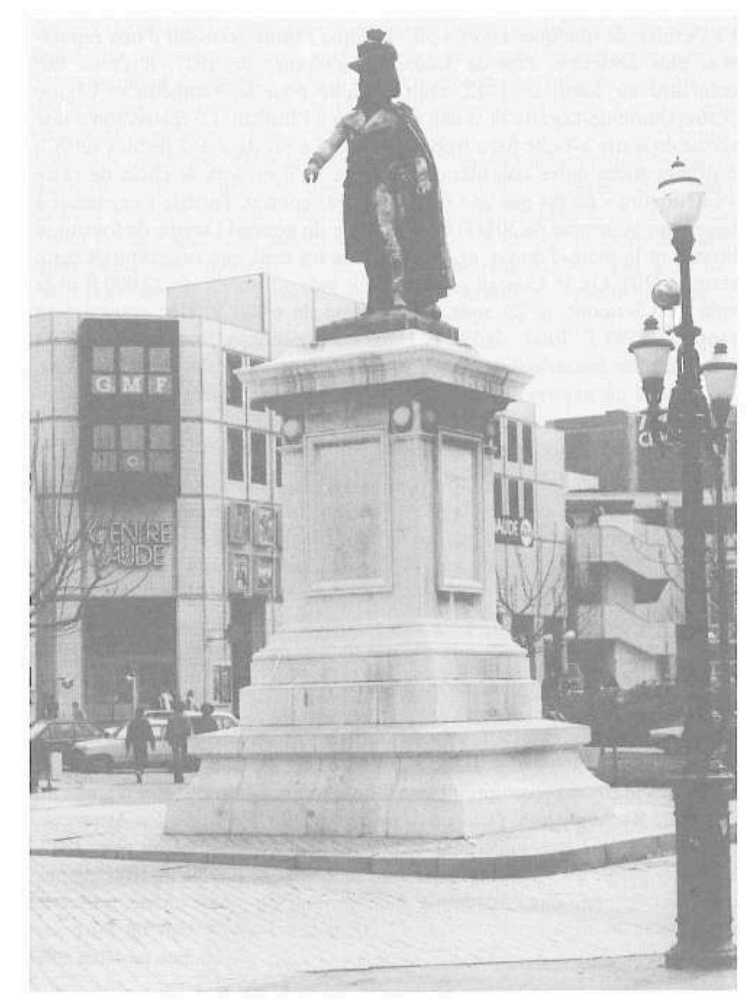

Fig. 2 - Nanteuil, Desaix, bronze, 1844 ; piédestal de 1903. Clermont-Fd., place de Jaude. État actuel. Photographie (c) (92) Inventaire général. S.P.D.E.M. Cliché Choplain-Masion.

Entre la commande et l'arrivée du monument à Clermont, Thiers avait commencé à publier l'Histoire du Consulat et de l'Empire (1845-1862), illustrée, dont le tome IV comprend une lithographie d'Alfred Revel d'après un dessin de Karl Girardet, Desaix à 
Marengo (Desaix à cheval). En Auvergne, le souvenir du héros auvergnat est ravivé par deux initiatives : en 1842, F. Maury publie à Clermont Desaix dans la Thébaïde, poème en trois chants, ouvrage couronné par l'Académie de Clermont. La même année, un jeune artiste clermontois, Louis Devedeux (1820-1874), qui venait de faire ses débuts au Salon de Paris (1838), entreprend dans son atelier parisien une grande peinture très colorée (2,495 x 1,662 mètres) qu'il donnera au musée de sa ville, Desaix en Égypte ${ }^{11}$.

Deux ans après, un avocat clermontois, Louis-Charles-Antoine Allemand, publie un Essai sur le général Desaix.

Le héros de Marengo peut revenir dans sa province natale, il y est attendu.

La Seconde République proclamée, le bronze de Nanteuil quitte enfin Paris. Reste à financer le piédestal qui supportera les $3200 \mathrm{~kg}$ de bronze.

Alors commence une longue et assez sordide aventure. Un dessin conservé aux archives communales de Clermont prévoit un piédestal de 5 mètres de haut, en pierre de Volvic. Le devis - matériau, maçonnerie, grille, marbre - s'élève à 5 567, 56 F. Or les sommes rassemblées couvrent péniblement le coût du bronze. Pour les cérémonies d'août 1848, la statue fut donc placée sur un piédestal « provisoire » - type d'aménagement dont on connaît l'habituelle durée - en brique, plâtre et bois, entouré d'une grille légère, déjà tombée deux ans plus tard. Il fallut attendre 1861 pour que ce piédestal soit, non pas remplacé, mais simplement "restauré », entouré d'une nouvelle grille et éclairé par deux réverbères (Fig. 3 et 4). Le haut piédestal que nous voyons aujourd'hui a été élevé en 1903, à l'occasion de l'implantation du Vercingétorix de Bartholdi sur la place de Jaude. Les deux héros auvergnats se font face, de part et d'autre de la grande place rectangulaire. La volonté d'harmoniser cet aménagement urbain conduit à construire, pour la statue de Desaix, un piédestal composé des mêmes matériaux que celui qui supporte Vercingétorix: granit, pierre de Ruoms, pierre de Comblanchien, plaques de marbre. De plus, afin d'équilibrer la hauteur des monuments, le piédestal de Desaix, pourtant déjà jugé trop haut en 1848, est surélevé : 6 mètres de haut, plus élevé que l'effigie du héros, au détriment de celle-ci ${ }^{12}$.

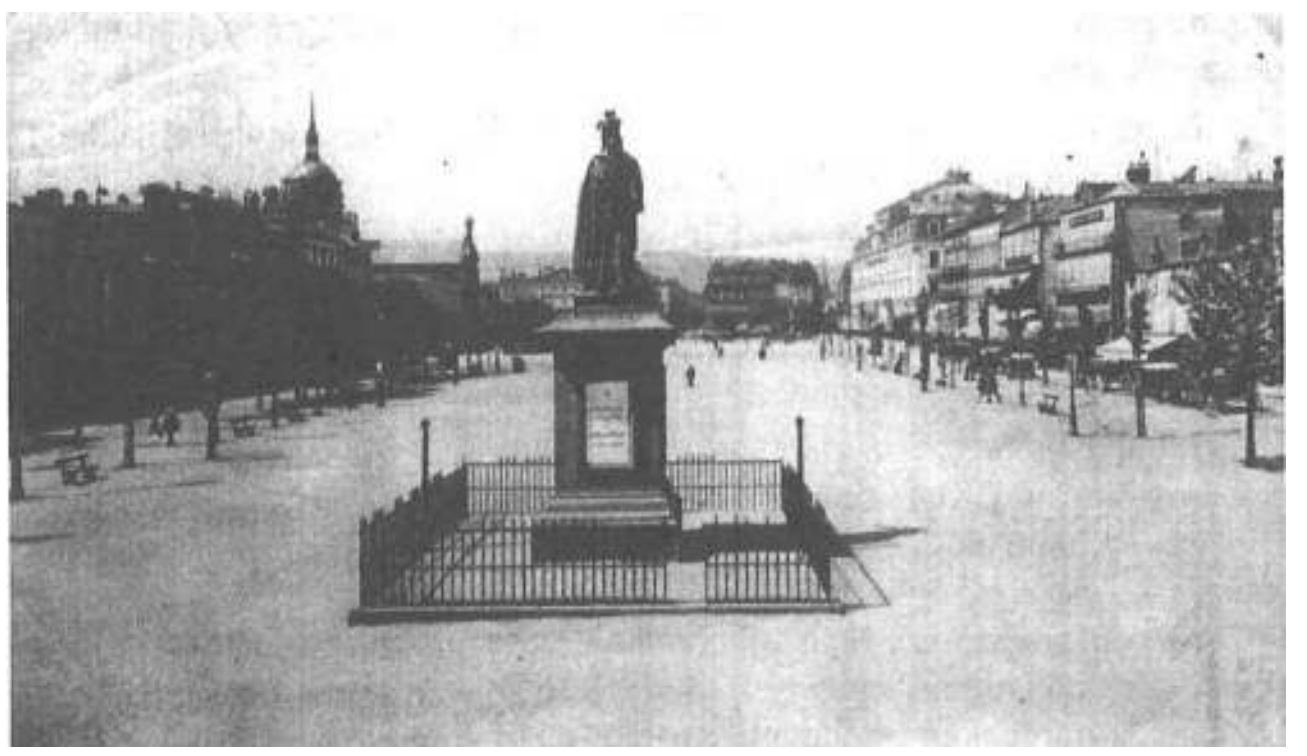


Fig.3 - La place de Jaude à la fin du XIXe siècle avec le monument à Desaix.Photographie ancienne, documentation Musées de Clermont-Fd.

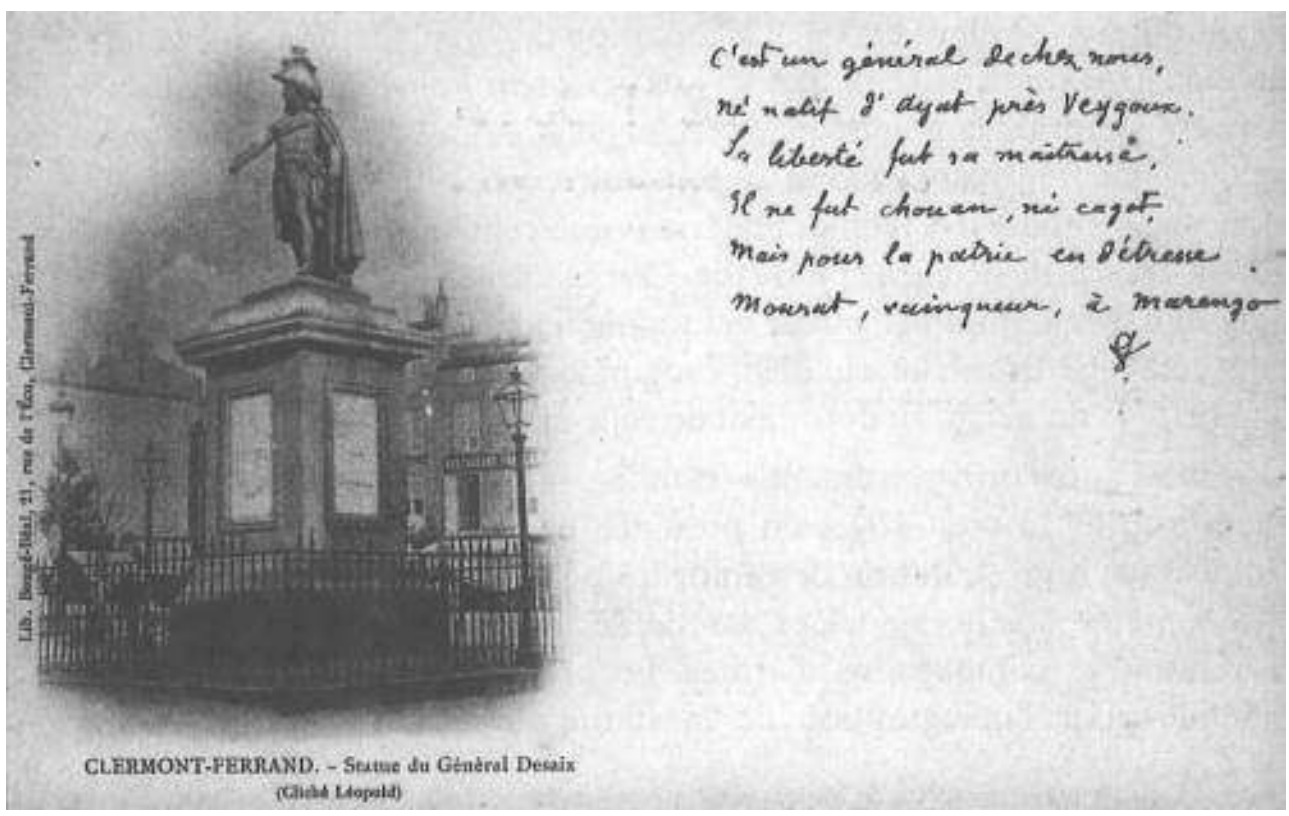

Fig. 4 - Statue du général Desaix, carte postale ancienne. Le texte manuscrit est édité avec la carte. Collection particulière.

Bien que «provisoirement » installée, la statue de bronze est inaugurée avec faste le 13 août 1848, en présence des autorités civiles et militaires - mais sans représentation des autorités ecclésiastiques, bien que les écoles chrétiennnes soient associées au défilé de la famille Desaix et des Vétérans, ses compagnons d'armes. Le programme de la «Fête départementale pour l'inauguration de la statue du général Désaix » [sic] ${ }^{13}$ précise notamment: "La place de Jaude sera décorée de mâts, de drapeaux et d'oriflammes, de faisceaux et d'emblèmes, rappelant l'époque et la gloire militaire de Desaix; l'épée du général, ses armes et l'habit d'uniforme qu'il portait à Marengo, seront exposés au pied de la statue.» Or, on sait qu'après sa mort Desaix avait été dépouillé de ses vêtements sur le champ de bataille, à l'exclusion de sa chemise, trop imbibée de sang. Sa longue et abondante chevelure avait permis à Savary de le distinguer dans l'obscurité parmi les nombreux cadavres. Mais l'authenticité d'une relique est-elle indispensable au culte?

À 11 heures, partant de la Préfecture et arrivant place de Jaude, un grand cortège parcourt la ville. Une salve de 10 coups de canon est tirée au moment du départ, une autre quand le statue est découverte. Suivent les discours, des musiques, des chœurs chantant des hymnes patriotiques, puis vient le défilé des troupes, ponctué de tirs de canon. Le soir, la place de Jaude et tous les édifices publics sont illuminés. À partir de six heures, des orchestres sont établis sur trois places de la ville pour des danses publiques. Il convient de noter que le programme signé du préfet, Dujardin-Beaumetz, demande aux «citoyens" (et non seulement aux habitants) de la place de Jaude de pavoiser leurs croisées et d'illuminer leurs maisons. "Tous les citoyens " sont invités à reconnaître l'autorité des Commissaires porteurs d'un brassard tricolore, chargés de veiller au détail de la fête. Car il s'agit bien d'une fête républicaine. Sur le monument étaient écrites les dernières paroles prêtées au héros, avec une variante notable: 
«Allez dire au Premier consul que je meurs avec le regret de n'avoir pas fait assez pour la République ». Et non plus : " pour la postérité ».

$\mathrm{Au}$ pied du monument s'élève la voix vibrante du jeune avocat clermontois A. Montander ${ }^{14}$ :

«Consolez-vous, ombres chères et sacrées, votre espérance et votre désir ne seront pas trompés. À côté du monument d'airain élevé à votre mémoire, nous en éleverons un autre plus digne de vous; nous fonderons la République que vous aimiez, comme vous l'aimiez, grande et pure comme votre gloire, immortelle comme votre souvenir, car nous la fonderons sur la triple base de la Justice, de l'Ordre et de la Liberté. »

Pourtant la devise de la République, qui figure sur le programme officiel, est bien «Liberté, Égalité, Fraternité ». Mais nous sommes en août, et non plus en février 1848. Après les journées de juin, le parti de l'Ordre a triomphé. L'effigie de la République, en statuaire, en monnaie, en gravure, bientôt en timbre, ne porte pas le rouge bonnet phrygien, elle ne tient pas les armes ni ne dévoile une généreuse poitrine. Chastement drapée, elle est couronnée de lauriers ou d'épis de blé15. Prudente République conservatrice qui conserve la Liberté mais remplace l'Égalité par l'Ordre et la Fraternité par la Justice !

Quoi qu'il en soit, l'ombre de Desaix n'a pas à se plaindre, ces années-là, de la postérité.

À l'occasion de l'inauguration de la statue, un artiste né à Clermont en 1820, MorelLadeuil qui travaillait à Paris depuis l'âge de 14 ans chez un fabricant de bronzes, revient s'installer de 1846 à 1848 à Clermont où il crée un grand médaillon de bronze (diamètre 16,6 cm) portant des inscriptions en creux : «À Desaix l'Auvergne 13 Août 1848 ». Signé à gauche : «L. Morel ». Marqué en bas : «Fod Barbier et Daubrée ». Sans égaler le vibrant Bonaparte de David d'Angers, ce portrait sensible, qui présente un Desaix un peu mélancolique, n'est pas sans mérite (Fig. 5).

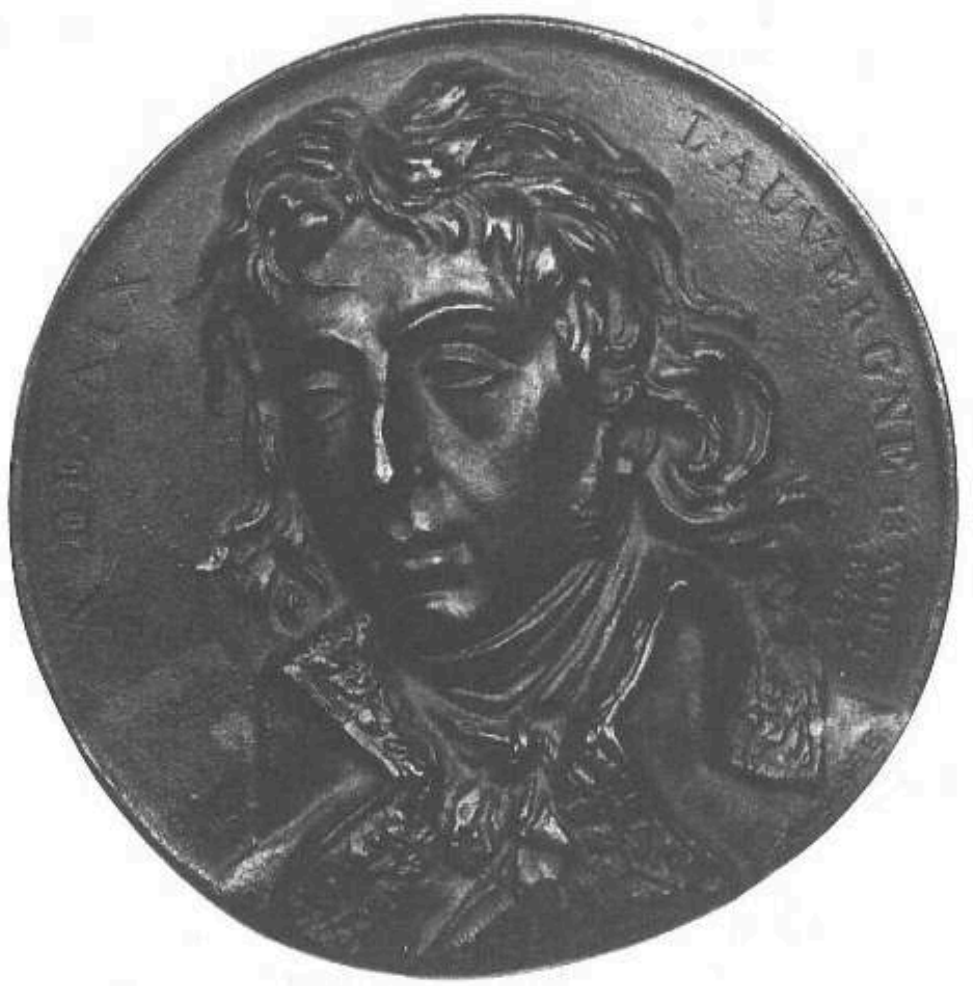

Fig. 5 - Morel-Ladeuil, À Desaix l'Auvergne, 1848, médaillon bronze, Musées de Clermont-Fd. 
31 Simultanément, en 1847, la commune de Combronde commande, par souscription, une fontaine «égyptienne » en pierre de Volvic au sculpteur volvicois Michel Channeboux. Le monument évoquant la campagne d'Égypte sera inauguré en 1849. Au-dessus d'un bassin circulaire s'élève une fontaine cubique. L'eau coule de la bouche du dieu Osiris. Au-dessus, sur un fût circulaire, sont sculptées deux gloires ailées qui tiennent des couronnes de laurier. Une sphère couronne l'ensemble. Deux plaques de marbre blanc portent des inscriptons gravées. Sur l'une, les victoires de Desaix: "Wissembourg. Kehl. Thèbes. Les Pyramides. Marengo.» Sur l'autre une variante des improbables dernières paroles de Desaix : «Allez dire au Premier consul que je meurs avec le regret de n'avoir pas assez fait pour la patrie et la postérité. » À la postérité s'ajoute la patrie, dans une formule qui honore l'esprit de synthèse des citoyens de Combronde

L'installation et l'inauguration $\mathrm{du}$ monument clermontois suscitent aussitôt la publication à Clermont d' une prolifération de textes :

33 - Anonyme, Chanson pour l'inauguration de la statue de Desaix; dédiée aux gardes nationaux du Puy-de-Dôme. - Brugiliolle, Vie de Desaix et Programme de la fête départementale pour l'inauguration de sa statue sur la place de Jaude à Clermont. - Hervier, Notice historique du général Desaix, Août 1848 - L'Inauguration de la statue de Desaix par une dame de Clermont. Adolphe Mazure, inspecteur de l'Académie de Clermont, Vie du général Desaix publiée à l'occasion de l'inauguration de sa statue sur la place de Jaude à Clermont-Ferrand, le 13 août 1848. - Pasteur J. Viguier, À Desaix. Pour l'inauguration de sa statue, à Clermont-Ferrand.

Bientôt paraît la première grande monographie de Desaix, écrite par F. Martha-Beker, Études historiques sur le général Desaix, Clermont, 1852. Après le décès de son oncle BagerBeker, beau-frère du général (qui avait épousé en 1800 Françoise des Aix), MarthaBecker prend connaissance des archives familiales, sur lesquelles il se fonde ${ }^{16}$.

Le Second Empire sera plus discret. Rien d'étonnant à cela : un autre héros, autrement prestigieux, occupe la scène. Toutefois, au Salon de 1857 figure une Mort de Desaix, peinture d'Eugène Ginain.

Desaix et l'esprit républicain

Une question a été posée au cours de ce colloque : Desaix était-il républicain ? Que la réponse soit positive, négative ou dubitative, une chose est sûre: s'il n'était pas républicain de son vivant, il l'est devenu dans la postérité. Desaix apparaît d'abord comme l'homme de la réconciliation. Sans doute est-il un militaire héroïque, un de «ces braves morts pour la patrie et pour la liberté » auxquels est élevée en 1800 une «colonne nationale»sur la place de la Révolution (actuelle place de la Concorde) ${ }^{17}$. Mais tel n'est pas son seul rôle.

Les premières manifestations en son honneur ont lieu à Paris à une date où la France souhaite en finir avec la Révolution. En 1800, la population aspire à la paix. Après Marengo, la victoire semble ouvrir une paix définitive à laquelle aspire la population et Bonaparte apparaît comme un faiseur de paix. Paix extérieure, paix intérieure aussi. La grande fête funèbre du Temple de Mars (les Invalides) est datée, selon le calendrier révolutionnaire, du 25 messidor. Ce 14 juillet « vieux style » n'évoque pas la prise de la Bastille. Au contraire, il s'agit d'une «Fête de la Concorde». Autant que Desaix, la spectaculaire cérémonie honore Turenne, le grand militaire de l'Ancien Régime, qui avait eu l'honneur exceptionnel d'être enseveli auprès des rois de France à Saint-Denis et dont le corps sera transféré en septembre aux Invalides ${ }^{18}$. Ces honneurs conjoints symbolisent, par une image forte, au moment où les émigrés commencent à rentrer en 
France, la volonté de réduire la fracture opérée dans la société par la Révolution. Déjà, l'année précédente, la grande peinture de David, l'Intercession des Sabines (1799, Musée du Louvre), exprimait ce souhait de voir cesser les luttes fratricides ${ }^{19}$.

Chronologiquement, le Desaix de 1848 est encore figure de transition, entre la monarchie de Juillet et la Seconde République. Mais sous la Troisième République Desaix va devenir une icône républicaine.

En 1888, des notables de Saint-Gervais d'Auvergne créent un comité pour l'érection, à Ayat-sur-Sioule, d'un monument commémoratif de ses campagnes. Arnaud, architecte à Clermont, élève une haute stèle de granit ornée d'un portrait de Desaix en bronze et gravée d'un long texte biographique. Le monument est entouré de quatre canons.

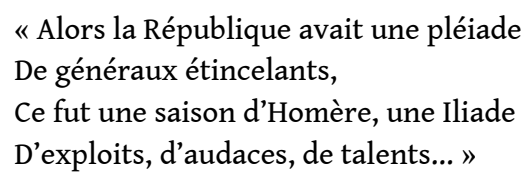

Ainsi commence l'ode qu'Emmanuel des Essarts, professeur à la faculté des Lettres de Clermont et auteur, en 1869, de Poèmes de la Révolution, avait composée pour l'inauguration, le 17 août $1890^{20}$.

41 Le même auteur composa une Ode triomphale récitée à Clermont devant le Desaix de Nanteuil, centre des grandes cérémonies des 30 juin et 1er juillet 1900 pour le centenaire de Marengo. Un haut portique en hémicycle, portant les noms des principaux faits d'armes de Desaix, avait été construit autour de la statue (Fig. 6). À l'exposition rétrospective des armées de terre et de mer, Étienne Clémentel, commissaire général de l'exposition ${ }^{21}$, faisant écho au portrait exalté que Michelet avait tracé de Desaix dans son Histoire de la Révolution, "Desaix, le plus pur de l'armée "22, célèbre « Celui qui fut le modèle le plus pur des héros de la République ». Ville pavoisée, défilé des troupes et des délégations, discours du général André, ministre de la Guerre... Les fêtes commémoratives sont résolument militaires et républicaines. Après la tentative du général Boulanger et l'Affaire Dreyfus, la Troisième République met en valeur le plus jeune général de la Première République. Autant qu'à Desaix, c'est à l'armée de la République que des Essart adresse ces vers :

"Ce flot envahisseur submergeait la Patrie,

Si l'Armée où toujours l'honneur étincela,

Impétueuse avec une noble furie,

N’avait répondu : "Halte-là !"

[Desaix tomba mort à Marengo]

Mais vivant dans les cœurs et dans la renommée

Il se dresse à nos yeux sur un civique autel,

Impérissable autant que la France et l'Armée

Mort vainqueur et mort immortel. $»^{23}$ 


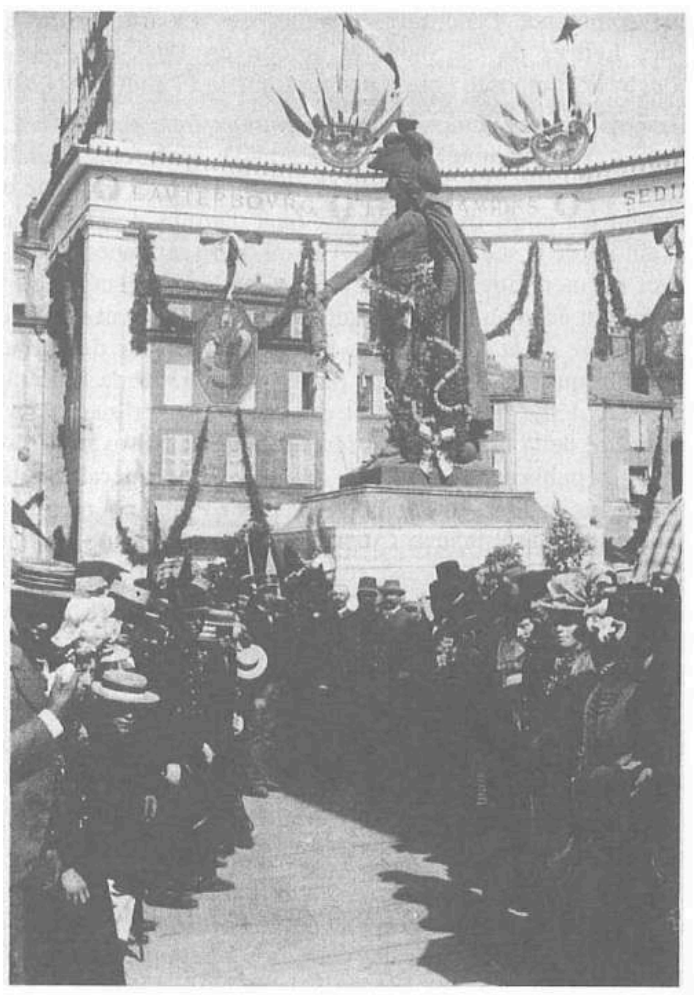

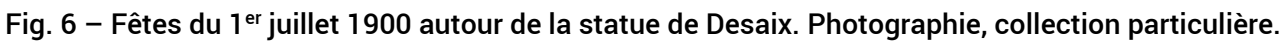

La même année 1900, le Conseil municipal de Riom sollicite de la ville de Paris l'envoi de la fontaine de Percier et Fortin, reléguée au dépôt des marbres depuis 1875 en raison des travaux d'agrandissement du Palais de Justice. Étienne Clémentel, maire de Riom, en obtient le don. Transportée à Riom, restaurée, elle fut inaugurée en 1906 lors de fêtes grandioses, subventionnées en partie par le Conseil général du Puy-de-Dôme ${ }^{24}$.

Et aujourd'hui ? Le monument de 1848 est toujours en place. Les plaques de marbre portent les inscriptions de 1903. Sur la face postérieure on peut lire: "Né à SaintHilaire d'Ayat le 27 août 1768 mort à Marengo le 14 juin 1800 ». Cette dernière inscription fait problème : la commune de Saint-Hilaire d'Ayat a changé de nom à la Révolution, pour devenir «Ayat-sur-Sioule». Le curieux qui chercherait aujourd'hui sur la carte, d'après cette inscription, le village natal de Desaix serait bien en peine de le trouver. Aussi la Ville de Clermont vient-elle d'accéder à la demande de la commune d'Ayat-sur-Sioule : placer sur la plaque, en surcharge, un bandeau gravé signalant le changement de nom.

Mais en dehors de cette anecdote, le monument de 1848 tient-il encore une place dans l'imaginaire collectif? Alors que le Vercingétorix de Bartholdi a encore joué au cours du xxe siècle un rôle de symbole de résistance à l'ennemi et de liberté d'expression ${ }^{25}$, le Desaix de Nanteuil est bien effacé.

Faut-il en chercher la cause dans le relatif oubli qu'a subi Desaix dans la première moitié du xxe siècle? Mais en 1954 Armand Sauzet publie Le Sultan juste ${ }^{26}$; en 1968 Ayat célèbre le bicentenaire de la naissance de l'enfant du pays. La mémoire de Desaix est revivifiée à la fin du siècle : exposition Desaix, général, au musée Bargoin de Clermont en 1983 ; en 1997 communication et exposition sur son voyage en Italie, à l'occasion du colloque sur La République directoriale ${ }^{27}$; la même année acquisition par la commune de 
Charbonnière-les-Varennes du manoir de Veygoux où Desaix avait passé son enfance ${ }^{28}$; en 1992 et 2000, restauration des deux monuments de Riom ; en 1998, de la fontaine de Combronde. Le bicentenaire de Marengo donne lieu à de nombreuses manifestations dans le Puy-de-Dôme : à Ayat-sur-Sioule, les 16 et 17 juin, en présence d'Alain Richard, ministre de la Défense ; à Effiat, le 23 juin ; à Riom, de janvier à juin, dont, en mai, le présent colloque. Mais rien ne s'est passé au pied du bronze de Nanteuil.

Ce désintérêt ne s'explique-t-il pas par la «médiocrité désespérante» de l'œuvre, aussitôt déplorée en $1848^{29}$ et la confusion de son environnement ? L'énorme bicorne plonge dans l'ombre, quelle que soit l'heure de la journée, un visage au demeurant sans expression. Le bras tendu doigt pointé vers le sol évoque plutôt un dresseur de chiens qu'un entraîneur d'hommes. Dominant à peine la mêlée, Desaix montre du doigt un hétéroclite champ de bataille: une esplanade encombrée de végétation, de lampadaires, de fontaines, de kiosques, de toiles de tentes... Une telle statue, raide, massive, insignifiante, évoque fort mal un personnage beaucoup plus complexe que les images hagiographiques ne le laissent penser.

\section{NOTES}

1.Monuments antérieurs à 1814 : fontaine par Laurent, Clermont-Ferrand, place du Taureau, 1801. - Fontaine par Percier et Fortin, Paris, place Dauphine, 1802 Cénotaphe de Strasbourg par Weinbrenner et Ohmacht, 1802. - Fontaine par Attiret, Riom, Pré-Madame, 1806. - Tombeau, Grand-Saint-Bernard, par Moitte, 1806. - Statue par Dejoux, Paris, place des Victoires, 1810.

2.Statue colossale en bronze, 1806-1810. Desaix y était représenté nu, appuyé sur un glaive, à côté d'un obélisque antique.

3.Ces dernières paroles attribuées à Desaix sont un chef-d'œuvre d'invraisemblance. Il faut du souffle pour articuler une telle phrase ! Comment pourrait-on la prononcer quand on vient de recevoir une balle en plein cœur? Et comment le témoin qui a vu tomber Desaix sans pouvoir le retenir, aurait-il pu entendre ces mots dans le fracas de la bataille ? Et à quoi aurait pu songer un officier à qui a été confiée une manœuvre de la dernière chance, sinon, à supposer qu'il soit encore en état de parler, à faire prévenir le général en chef que l'offensive souhaitée est en cours ? Et pourquoi appeler ce dernier par son titre civil ? Paroles fabriquées, comme tant de paroles « historiques ». 4.Cénotaphe du général Desaix, Strasbourg. Cénotaphe élevé par souscription par les soldats de l'armée du Rhin, 1802. Par l'architecte Weinbrenner et le sculpteur Ohmacht. À l'origine placé dans l'île des Épis, formée par le Rhin et le " petit Rhin ", il a été été transféré, en 1960, dans Strasbourg même, place du Maréchal-de-Lattre-de-Tassigny. 5.Le souvenir de Desaix est cependant présent dans l'espace urbain riomois, non seulement par les deux fontaines en son honneur, celle d'Attiret, 1806, et celle de Percier et Fortin, depuis 1906. Son nom a été donné successivement à plusieurs voies, au gré des fluctuations politiques. D'abord en 1864 à la rue de l'Hôtel-de-Ville. Mais en 1870 , la rue reprend son nom et Desaix remplace Eugène Rouher dans une rue voisine. En 1908 enfin, l'ancienne rue Desaix devient rue Gilbert-Romme et le grand boulevard 
du Collège devient boulevard Desaix. Desaix figure en outre dans les collections du Musée Mandet avec Mort de Desaix à Marengo, peinture de Regnault, 1801, dont il existe deux autres exemplaires, à Versailles et au Musée d'Art Roger-Quilliot de Clermont ; et un Portrait de Desaix par La Foulhouse, 1862.

6.La statue de Louis XIV par Bosio

7.Conseil général, 1839, Procès-verbaux. Minutes. Archives départementales du Puy-deDôme. [1848 : cote N 0253].

8.Le monument sera restauré et complété en 1903 par Poncelet, architecte de la ville de Clermont. Ces modifications font partie du plan d'embellissement de Clermont à l'occasion de l'inauguration de la statue de Vercingétorix.

9.Après cet échec, les succès d'Antonin Moine ont pris fin. Il se suicidera en 1849.

10.Le devis de Bartholdi pour son Vercingétorix ne s'élèvera, soixante ans plus tard, qu'à $35000 \mathrm{~F}$.

11.Ainsi que la maquette de Moine, le tableau, conservé au Musée d'Art Roger-Quilliot, a figuré à Riom au Musée Mandet, d'avril à septembre 2000, à l'exposition Desaix, images et témoignages. Devedeux ne sera que peu peintre d'histoire. Mais il trouve ici l'occasion, en célébrant un héros national et auvergnat, de faire œuvre d'orientaliste. Car sa principale source d'inspiration, c'est l'Orient bien qu'il n'y soit jamais allé, mais il se fonde sur les récits de voyage, les dessins, les estampes, les documents rapportés par les voyageurs.

12.Archives communales de Clermont, M 199.

13.Fête départementale pour l'inauguration de la statue du général Désaix. Programme. 1er août 1848. Archives départementales du Puy-de-Dôme. 6 BIB 659. Dans les textes de 1848 , le nom de Desaix est orthographié « Désaix », avec un accent aigu sur le e. Ce que ne feront ni Martha-Becker en 1852 ni les textes de 1900. Comment convient-il donc de prononcer le nom du général ? Actuellement, en Auvergne, l'usage est de prononcer de $z a i$. Le $x$ final des noms ne se prononce jamais. L'orthographe patronymique des ancêtres du général a varié depuis le XVIII ${ }^{\mathrm{e}}$ siècle : des Aix, des Haies, des Ayes... Le nom du jeune Louis est écrit « Desaix de Veygoux » sur les registres de l'École royale militaire d'Effiat. Le certificat de noblesse établit par Bernard Chérin orthographie « des Aix » le nom de Louis et de ses ancêtres. Le comité de surveillance de Riom dénonce, en 1793, « le nommé Désaix ». Lui-même a parfois signé « des Aix » ou « Des aix ». Puis toujours « Desaix », sans accent sur le $e$, qu'il écrive à sa sœur Françoise ou à Bonaparte. Mais jamais avec deux $s$ à la suite. On pourrait donc hésiter entre dé zai et de $z a i$. Mais le $s$ est toujours dur. La graphie avec deux $s$ apparue dans certains textes du $\mathrm{XIX}^{\mathrm{e}}$ siècle, ne se justifie pas. Voir sur le même sujet, dans ce volume, les observations de Philippe J. Vidal.

14.Alain Montander (1815-1867), avocat né à Clermont, ami d'enfance d'Eugène Rouher. Voir : Georges Bonnefoy, Histoire de l'administration civile de la province d'Auvergne, t. III, Paris, 1902, pp. 250 et 829.

15.Voir : Maurice Agulhon et Pierre Bonte, Marianne, Les visages de la République ;

Gallimard, 1992.

16.Toujours utile, notamment pour les documents reproduits en fin d'ouvrage, cette publication doit cependant être consultée avec précaution.

17.Une erreur souvent reproduite confond cette colonne éphémère de Moreau (en bois et plâtre) de 1800 avec la colonne Vendôme (1806).

18.Voir : Bronislav Baczko, «Turenne au temple de Mars », Guerre et Paix. Mélanges offerts à Jean-Claude Favez, Genève, 2000, pp. 75-89. 
19.Le titre exact de la peinture est bien l'Intercession des Sabines, et non pas leur « enlèvement », erreur obstinément répétée, jusque dans les ouvrages par ailleurs les plus sérieux. Sans lire le livret dans lequel David avait expliqué le sujet de son tableau, il n'est qu'à regarder celui-ci. On y voit des femmes qui s'interposent entre deux combattants affrontés en présentant leurs enfants, fils ou neveux des uns ou des autres. Il y a beau temps que les Sabines ont été enlevées... Mais ceci est moins excitant. 20. «e monument à Desaix », Revue d'Auvergne, 1890, pp. 416-417.

21.Étienne Clémentel (1864-1936). Député républicain du Puy-de-Dôme en 1900, sénateur en 1920, plusieurs fois ministre de 1905 à 1924. Maire de Riom de 1904 à 1935. En collaboration avec des Essarts, Clémentel a composé en 1900 un "drame lyrique », La Mort de Desaix, qu'il ne réussit pas à faire jouer à Paris. Inédit. Manuscrit conservé aux Archives départementales du Puy-de-Dôme (Fonds Clémentel).

22. Michelet, Histoire de la Révolution française, livre VI, chapitre IX, édition de la Pléiade, 1961, vol. I, p. 925. Michelet écrit encore : «C'était l'homme de sacrifice qui voulut toujours le devoir, et la gloire pour lui jamais, qui la donna souvent aux autres, et même aux dépens de sa vie, un juste, un héros, un saint, l'irréprochable Desaix ». 23. "Ode prononcée à Clermont pour le centenaire de Desaix », Revue d'Auvergne, 1900, pp. 235-238.

24.Voir le catalogue de l'exposition Louis Charles Antoine Desaix, général, par Antoinette Ehrard et Gérard Tisserand, Musée Bargoin, Clermont-Ferrand, 1983. Numéros 94 à 102. 25.Antoinette Ehrard, "Vercingétorix contre Gergovie », Nos ancêtres les Gaulois, Actes du colloque international de Clermont-Fd, recueillis par Paul Viallaneix et Jean Ehrard. Publications de la Faculté des lettres et de sciences humaines de l'Université de Clermont-Fd. II, Nouvelle série, fascicule 13, 1982, pp. 307-320.

26.L'ouvrage de Sauzet, Le Sultan juste, 1954, est plus fiable que celui de Martha-Beker. Malheureusement, l'auteur fait référence à des documents qu'il a pu personnellement consulter dans une collection privée qu'il n'a pas eu l'autorisation de localiser. L'ouvrage récemment paru, Le sultan de Bonaparte, de Gonzague Saint-Bris, n'apporte rien de nouveau.

27.Antoinette Ehrard, « Desaix en Suisse et en Italie, 1797 », La République directoriale, Actes du colloque de Clermont-Fd, Société des études robespierristes. Centre d'histoire des entreprises et des communautés. Centre de recherches révolutionnaires et romantiques. 1998, Tome 2, pp. 921-933.

Sur les pas du général Desaix en Suisse et en Italie, 1797, brochure d'accompagnement de l'exposition (extraits annotés du « Journal » de Desaix, illustrations noir et couleur), Service Universités-Culture, Clermont-Fd, 1997.

28.Louée au Syndicat mixte pour l'aménagement et le développement des Combrailles (SMADC), la demeure, très transformée, est le lieu d'un « scénomusée » évoquant, de façon très libre, la vie de Desaix.

29. " Médiocrité désespérante ", " pose guindée et prétentieuse », Gazette d'Auvergne et du Bourbonnais, 1er août 1848. « Lourdeur désagréable à l'œil », " geste d'un magister irrité », J. Bonneton, Bustes et sculptures de Clermont, 1909. 


\section{RÉSUMÉS}

À Clermont-Ferrand, place de Jaude, le visiteur peut découvrir aujourd'hui, tout près du monumental et fougueux Vercingétorix de Bartholdi, une modeste statue en bronze de Desaix. Bien qu'inaugurée en août 1848, elle n'est pas d'origine républicaine : c'est dix ans plus tôt que le Conseil général du Puy-de-Dôme en avait arrêté le principe, dans le cadre de la politique de réconciliation nationale voulue par le roi des Français, politique qui mettait l'accent, notamment dans les commandes publiques, sur la continuité de l'Histoire de France. La mémoire de Desaix avait bénéficié de ce mouvement, dans les arts comme dans les textes. Le choix du projet de Nanteuil, préféré à celui d'Antonin Moine dont le musée R. Quilliot de Clermont conserve la maquette, s'explique peut-être par la notoriété officielle d'un ancien Prix de Rome, entré depuis peu à l'Institut; peut-être aussi par des raisons d'économie : ici le héros est à pied, et non à cheval comme celui de Moine. Quoi qu'il en ait été, le financement de la commande devait être difficilement assuré, et la réalisation n'emporta pas plus l'adhésion des contemporains qu'elle ne réussit à forcer la nôtre. L'inauguration fut pourtant une grande fête républicaine. Et en 1900 la statue sera au centre des cérémonies du centenaire de Marengo : le besoin où sera alors la IIIe République du prestige d'un général républicain explique l'honneur fait à un monument médiocre qui ne revivra jamais cet instant de gloire ni au long du xxe siècle, ni en juin 2000.

Concerning the Statue of Desaix by Nanteuil in the Place de Jaude at Clermont-Ferrand.

At Clermont-Ferrand, in the place de Jaude, the visitor to-day discovers, close to the monumental and fiery Vercingetorix by Bartholdi, a modest bronze statue of Desaix. Although inaugurated in August 1848, it is not of republican origin. Ten years earlier, the General Council of Puy-de-Dôme had decided to erect one, in the context of the policy of national reconciliation ordained by the King of the French, a policy that highlighted the continuity of French history wherever public commissioning was involved. The memory of Desaix benefited from this trend in art and letters. The choice of Nanteuil's project, preferred to that of Antonin Moine, whose model is conserved at the R. Quilliot Museum in Clermont, is probably due to the public notoriety of a former Prix de Rome, recently admitted to the Institute. Also perhaps to reasons of economy, for here the hero is on foot, not on horseback as in the Moine version. Be that as it may, the funding of the project was no easy task and the result did not inspire more admiration then than it does to-day. The inauguration, however, was a festive republican event. And in 1900, the statue occupied centrestage in the ceremonies marking the Marengo centenary. The need felt by the Third Republic for the prestige of a republican general explains the honour showered on a second-rate monument which never relived that moment of glory throughout the 20th century, let alone in June 2000.

INDEX

Mots-clés : Marengo, République, Bartholdi, Moine, Nanteuil

\section{AUTEUR}

\section{ANTOINETTE EHRARD}

Université Blaise-Pascal. Centre de Recherches Révolutionnaires et Romantiques 